\title{
Repensando la noción de 'comunidad' en contextos de alta migración: el caso de Pepinales
}

Jacques Ramírez ${ }^{1}$

Nunca la palabra "comunidad" se había utilizado de modo más indiscriminado y vacio que durante las décadas en las que las comunidades, en sentido sociológico, se convirtieron en algo difícil de encontrar en la vida real.

(Eric Hobsbawn)

Resumen

El PRESENTE ARTíCULO TIENE COMO OBJETIVO CENTRAL RASTREAR Y REPENSAR EL CONCEPTO DE 'COMUNIDAD' EN contextos de alta migración. A partir de un trabajo etnográfico realizado sobre Pepinales, el cual ha EXPERIMENTADO FLUJOS MIGRATORIOS INTERNOS E INTERNACIONALES POR MÁS DE CUATRO DÉCADAS, CUESTIONAMOS LAS NOCIONES ClÁSICAS dE COMUNIDAd ASí COMO SEÑALAMOS LAS LIMITANTES dE LAS PROPUESTAS DE LOS ESTUdIOS MIGRATORIOS AL HABLAR DE 'COMUNIDADES TRANSNACIONALES' Y EL USO DEL TÉRMINO 'COMUNIDADES FANTASMAS'. LUEGO DE REALIZAR UN RECORRIDO TEÓRICO POR ESTE CONCEPTO, PROPONEMOS VER A LAS COMUNIDADES, EN CONTEXTOS DE ALTA MIGRACIÓN, COMO RE-DESTERRRITORIALIZADAS FORMANDO UN ARCHIPIÉLAGO ACTIVO EN MOVIMIENTO.

Palabras Claves: comunidad- migración-transnacionalismos- Redes-ARChipiélago.

Abstract

THE OBJECTIVE OF THE PRESENT ARTICLE IS TO TRACE AND RETHINK THE CONCEPT OF "COMMUNITY" IN CONTEXTS of high migration. Through ethnographic work carried out in Pepinales, an area that has undergone INTERNAL AND INTERNATIONAL FLOWS OF MIGRATION FOR MORE THAN FOUR DECADES, WE QUESTION CLASSIC NOTIONS OF COMMUNITY AND POINT OUT THE LIMITATIONS OF THE PROPOSALS OF MIGRATION STUDIES UPON DISCUSSING “Transnational COMmunity," AND the USE OF THE TERM "GHOST COMMUNities." AFter COVERING THE THEORIES OF THIS CONCEPT, WE PROPOSE TO SEE COMMUNITIES IN HIGH MIGRATION CONTEXTS AS RE-DE-TERRITORIALIZED FORMING AN ACTIVE ARCHIPELAGO IN MOVEMENT.

KEYWORDS: COMMUNITY -MIGRATION -TRANSNATIONALISM-NETWORKS-ARCHIPELAGO.

1 Doctor en Antropología Social, Universidad Iberoamericana, México, D.F. Director del Instituto de la Ciudad, Quito. Profesor-investigador asociado FLACSO-ECUADOR. papodelalife1@yahoo.es. 


\section{Introducción}

U no de los conceptos claves dentro de las ciencias sociales ha sido el de "comunidad". Desde los orígenes de las ciencias sociales, pensadores como Rousseau, Vico, Maine, Morgan, Marx, Weber, utilizaron dicha noción al momento de analizar las particularidades específicas de las que cada uno se ocupó.

Desde aquella época hasta la actualidad, la noción de comunidad ha seguido rondando los pasillos de la academia -y fuera de ella-, sin embargo, su uso ha sido ambiguo, poco riguroso y polisémico, tratando con él de explicar varias cosas a la vez. Zárate (2005:61), retomando a Hunter y Whitten, señala que el término comunidad ha sido usado con gran liberalidad por los sociólogos, para caracterizar una amplia gama de grupos cuyos intereses respectivos comparten un sentido de identidad, valores e intereses específicos, y una definición de funciones o papeles sociales concretos con respecto a los demás. En este sentido general, un poblado, una vecindad, una sociedad recreativa, un sindicato obrero o una profesión colegiada pueden entenderse como comunidad.

Con el paso del tiempo y el continuo aumento de la especialización dentro de las ciencias sociales, se empezó a dar más importancia al adjetivo calificativo que acompañaba a la noción de comunidad quedando esta en un segundo plano, o dando por sentado y sin mayor cuestionamiento el significado del mismo. Así surgieron los estudios de comunidades agrícolas, campesinas, étnicas, migrantes, etc.

El objetivo del presente artículo es, por un lado, rastrear la noción de comunidad que ha utilizado la antropología clásica haciendo un ejercicio de aterrizaje en el uso de este concepto dentro de la academia ecuatoriana y, por otro, rastrear los aportes de los estudios transnacionales con énfasis en la noción de comunidad transnacional. Luego de pasar revista algunos de los trabajos realizados en Ecuador, y presentar una breve información y descripción de la comunidad de Pepinales, se cierra el texto con la propuesta de entender a las comunidades, en los contextos de alta migración, como re-desterritorializadas formando un archipiélago activo en movimiento.

Este ejercicio teórico se basa en un trabajo etnográfico, algo que cada vez se hace menos, del pueblo de Pepinales del cual se describe brevemente sus flujos migratorios internos e internacionales ${ }^{2}$. Consideramos que analizar las especificidades locales permite someter los conceptos a un proceso de reelaboración o reconstrucción, a fin de poder captar e interpretar de manera más cabal dichas realidades.

\section{Rastreando a la 'comunidad'}

Si bien la noción de comunidad ha estado presente prácticamente desde el surgimiento de las ciencias sociales, fue el alemán Ferdinand Tönnies quien en el siglo XIX puso este concepto en el centro de la mesa de debate al publicar el libro "Comunidad y Asociación" en 1887.

Este autor plantea la diferenciación entre la comunidad o gemeinschaft y la asociación o gesellschaft que constituyen maneras opuestas de construir la vida en sociedad. La primera "constituye la forma genuina y perdurable de la convivencia", mientras que la segunda es transitoria y superficial. La gemeinschaft se constituye de manera natural y su voluntad es esencial, mientras que la gesellschaft es racional y su voluntad es arbitraria e instrumental" (Tönnies en Lisbona, 2005: 27).

2 La etnografía histórica de Pepinales fue lo que le dio sustento a mi tesis doctoral titulada "Dónde esta la comunidad? la migración ecuatoriana a Alemania y España. El caso de Pepinales". 
En este sentido, para el pensador alemán “allí donde los seres humanos están relacionados por voluntad propia de una manera orgánica y se afirmen entre ellos, encontraremos una u otra forma de comunidad" (Delgado, 2005: 40). En palabras del autor alemán, la comunidad como Gemeinschaft se expresa en la vida en familia en concordia, en la vida del pueblo rural, en la cultura popular, y en la vida de ciudad en la religión (Tönnies, 1979: 231). Igualmente señala dicho autor que uno de los requisitos para que exista comunidad es la interacción cara a cara y un territorio (pueblo, barrio, etc.) en el que ésta pueda ocurrir.

Estas ideas con cierto aire idílico de Tönnies fueron retomadas por la antropología clásica, constituyéndose la comunidad en el 'objeto’ central de estudio y el lugar donde realizar en trabajo etnográfico. Así, al hablar de comunidad se pensó en aquellos pequeños poblados campesinos/ rurales que se refieren a una entidad claramente diferenciada y definida espacialmente en términos de su localización y características culturales particulares. Los trabajos de Redfield son un buen ejemplo de lo señalado.

Dentro de la teoría social pensadores como Durkheim y Weber siguieron la línea evolutiva propuesta por Tönnies. Por un lado, Durkheim en su libro "La División del Trabajo Social"3 hace una distinción entre dos tipos ideales de sociedad. Habló de solidaridad mecánica donde Tönnies habló de comunidad ${ }^{4}$. En este estado primitivo se da poco o nula división del trabajo en oposición a las sociedades modernas donde existe una solidaridad orgánica y donde hay una mayor y más refinada división del trabajo. Delanty (2006) señala que en Durkheim la idea de comunidad se ve como una fuerza moral para la modernidad ya que el pensador francés creía que "las normas básicas del individualismo moral ya se encontraban en las formas orgánicas de la solidaridad que emergen con la división del trabajo en la sociedad" (Íbid: 56-57).

Por su parte, para Weber (1984), la relación social denominada comunal se inspira en el sentimiento subjetivo de los participantes de construir un todo y, por el contrario, la relación social denominada sociedad o asociativa se inspira en una compensación de intereses por motivos racionales.

Visto así, el concepto de comunidad, desde la modernidad surge como opuesto a sociedad dentro de un esquema evolutivo. En tal sentido, la noción de comunidad se asocia con ideas de 'retraso', 'inferioridad', 'no racional', como opuesto al proyecto moderno que amenaza al modelo capitalista. Igualmente, la comunidad -desde esta visión romántica- se ve como algo "puro" que se está perdiendo, algo que esta desapareciendo.

Posteriormente, este modelo binario societal, uno inferior, arcaico, pre moderno, subjetivo y otro superior, moderno, racional se solidificó en la escuela de Chicago ${ }^{5}$ de la mano de un antropólogo que, a la larga, tendría gran repercusión en la antropología latinoamericana: Robert Redfield. Dicho autor, a partir de sus trabajos etnográficos realizados en México, desarrolla el

3 Que constituyó la tesis doctoral publicada por primera vez en 1893 y según algunos autores revocó los postulados de Tönnies.

4 Aunque algunos autores señala que existe una aparente inversión de sentido entre la opinión de Durkheim y Tönnies. El primero habló de solidaridad mecánica donde el segundo habló de comunidad, y de solidaridad orgánica donde Tönnies habla de sociedad. Para Delgado (2005) la solidaridad mecánica no corresponde a la gemeinschaft ni la orgánica a la gesellschaft. Dicho autor señala que "este malentendido es sistemático pero en realidad poco tienen en común a no ser una misma secuencialización en un determinado sentido evolutivo de dos grandes tipos de sociedades, que hace que unas -la gemeinschaft y la basada en una solidaridad mecánica- precedieran históricamente a las otras. Sin embargo, existe un diferencial teórico fundamental entre dos sugestiones teóricas que, de forma precipitada, se han tendido a equiparar" (Delgado, 2005: 45-50).

5 Como señala Delgado (2005: 42) "dirigiendo su mirada a las sociedades de origen de los inmigrantes, los teóricos de Chicago quisieron ver en ellas la vigencia en otros sitios de ese modelo integrado y pacífico de sociedad a pequeña escala, en que podía encontrarse todo lo que la sociedad urbana no podía ofrecer: una convivencia en que se respetaba el pasado, cuyos componentes se sentían vinculados a través de poderosos sentimientos de pertenencia identitaria y, sobre todo, una sociedad consecuente consigo misma, en que cada lugar estructural esta coherente con todos los demás y con su visión del universo y en la que cualquier amenaza para esa congruencia al tiempo social y cósmica era rápidamente neutralizada". Cabe señalar que dichas amenazas para los pensadores de Chicago provenía de la comunicación y contacto con el exterior y ajena a la comunidad. 
concepto el 'continum folk urbano'. En estos trabajos el autor establece las características de una comunidad -singularidad, tamaño pequeño y autosuficiencia- a partir de su trabajo en Tepoztlán (1930) y su evolución que conduce de una sociedad campesina arcaica folk como es Tusik a otra evolucionada, moderna, urbana como Mérida en la Península de Yucatán (1940)

Dentro de este modelo evolutivo quedó sentado que los grupos indígenas campesinos y rurales estaban en la escala menor y como señaló el antropólogo mexicano Aguirre Beltrán (1973: 156), dichas comunidades constituían "sociedades sin clases sociales, organizadas como un grupo de status, de índole sacra y naturaleza folk".

Esta forma de entender la realidad fue paradigmática por mucho tiempo dentro de la disciplina antropológica $^{7}$ (incluso en la actualidad goza de buena aceptación) lo que condujo a la tarea titánica de los antropólogos en búsqueda de ‘comunidades en estado puro’ y sin o poco contacto con la sociedad mayor y en otros casos se empezó a estudiar los cambios y las continuidades que se ha producido a lo largo de los años.

Esta corriente de pensamiento conocida como funcional-culturalista que definió a la comunidad en términos esencialistas, naturales, cerradas y ahistóricas, se introdujo a la academia ecuatoriana a partir de los vínculos y semejanzas que ciertos antropólogos vieron entre las comunidades campesinas/indígenas de México y Ecuador.

La influencia del indigenismo mexicano en Ecuador fue tan notoria que contribuyó a delimitar la agenda investigativa y curricular de la naciente disciplina antropológica desde la década del 70. Como señala Martínez (2007: 4), "grandes pensadores mexicanos como Moisés Saenz, José Vasconcelos, Gonzalo Aguirre Beltrán, Guillermo Bonfil, y otros visitaron el Ecuador e influyeron a través del Instituto Indigenista Interamericano en las políticas públicas ecuatorianas.

Aparte de los aportes de la antropología mexicana, los estudios en Ecuador se vieron influenciados por dos vertientes teóricas diferentes: los estudios de Chayanov y en general de los trabajos sobre la viabilidad de la comuna rusa y los escritos de Mariátegui sobre la potencialidad política de la comunidad andina en el Perú (Martínez, 2002). Estas perspectivas tienen una visión más integral y dan cuenta del campesinado y su lugar dentro del sistema capitalista.

En este contexto, la antropología ecuatoriana desde sus orígenes se preocupó por estudiar fundamentalmente las comunidades indígenas de la sierra y oriente ecuatoriano ${ }^{8}$ que atravesaban -desde la década del sesenta- por un proceso de reforma agraria. Planteaban la ecuación de entender la comunidad como una forma de organización social más territorio. Algunos campesinistas como García (1980) pensaban que el capitalismo estaba erosionando los tipos de solidaridad tradicional 'propias' de los indígenas como la reciprocidad, el parentesco y el trabajo comunal (la minga) y que la migración del campo a la ciudad se veía como la principal forma de articulación con el capitalismo y de destrucción de éstas formas tradicionales de resistencia (Sánchez Parga, 2002).

Como se ha dicho, casi la totalidad de estos trabajos se enfocaron en estudios de comunidades indígenas y sus prácticas culturales: "rescataron la comunidad andina y sus tradiciones históricas como estrategias de resistencia al avance del capitalismo que debían ser promovidas por aquellos que buscaban el bienestar campesino y su viabilidad como grupo después de la Reforma Agraria" (Martínez, 2007).

6 Años después publicó The Little Commnunity (1955). Para aquel entonces ya se acentuó la forma dicotómica de analizar la sociedad vistas como folclórico/urbano, tradicional/moderno, pequeña tradición/gran tradición, etc.

7 Y sociológica. Cabe recordar que Parsons (1951) definió a la comunidad como aquella colectividad cuyos miembros comparten un área territorial común como base de operaciones para las actividades diarias.

8 Existen muy pocos trabajos de comunidades campesinas mestizas, uno de ellos es el de Stolen (1987). Casi todos se centraban en estudios de comunidades con población indígena, pero lo 'étnico' era y es analizado por lo general en términos esencialistas a partir del estudio de variables como la lengua, el vestido, las costumbres y la religiosidad. Cabe indicar, como señala Luciano Martínez (2002) que el tema de las comunidades indígenas fue preocupación de pensadores liberales desde principios y mediados del siglo XX. 
Cabe señalar sobre este último aspecto que fueron los estudiosos de antropología religiosa andina quienes buscaron nuevos marcos interpretativos para entender la comunidad sobre todo en base a los trabajos de Turner para quien la comunidad tiene un carácter simbólico en el sentido de que crea vínculos poderosos entre los miembros de una sociedad o de un grupo social. Para dicho autor, la comunidad se entiende mejor como communitas para distinguir una particular forma de relación social que existe en todos los tipos de sociedad y que no puede reducirse a la comunidad en el sentido de una agrupación fija y específica en un espacio.

Es desde finales de los ochenta que surge una corriente de estudios antropológicos de la globalización o el transnacionalismo que han sido muy críticos no solamente con el uso de ciertos conceptos clásicos de la antropología como cultura, identidad o comunidad, sino también en la forma tradicional del quehacer etnográfico.

Autores como Appadurrai (1996), Clifford (1999) o Gupta y Ferguson (1997), han dado nuevas pautas para ver la relación entre cultura, personas y espacio para 'despurificar' o 'desnaturalizar' dicha relación a partir del análisis de la intensificación de los flujos de personas, dinero, ideas, objetos, información, imágenes, etc., que cruzan fronteras. Desde esta perspectiva, vivir en un mundo interconectado implica repensar la noción de comunidad la cual la entienden como un lugar particular, diferenciado y opuesto a otros lugares, construido políticamente a través de prácticas incorporadas donde lo comunal y lo comunitario no es solamente el reconocimiento de una similitud cultural o de una contigüidad social, sino un sistema de clasificación que está fundado en varias formas de exclusión y de construcción de la 'otredad' que definen las identificaciones personales y de colectivo (Gupta y Ferguson, 1997:13).

Si bien esta última perspectiva es útil y ha sido retomada por varios autores de la migración, considero que poner el énfasis en las interconexiones y el flujo de bienes de diferente índole hizo perder de vista los lugares y sobre todo las personas que dan vida a esas interconexiones. Esta perspectiva que cobra fuerza en los noventa, puso el énfasis en el tema de las redes, circuitos, espacios o campos trasnacionales para resaltar las relaciones sociales que traspasan fronteras. Expliquemos más detenidamente esta visión.

\section{Comunidades transnacionales y migración}

Los orígenes del enfoque trasnacional surgen a principios de la década de los 90's a partir de ciertos trabajos como los de Rouse (1991), Kearny (1991), Goldring (1992), Glick Shiller et. al. (1992) estas últimas a partir del denominado transnational approach definen el transnacionalismo como "el proceso por el cual los inmigrantes construyen, forjan y sostienen campos sociales y relaciones sociales multi-situadas que unen su país de origen con el de destino" (1992:10).

Aunque cada autor dentro de esta perspectiva ha tratado de dar su concepto de transnacionalismo ${ }^{10}$, en términos generales se utiliza esta noción, en la dirección señalda anteriormente, para referirse a la existencia de sólidos nexos entre los lugares de origen y destino de los migrantes. La especificidad de estos nexos es que requieren de contactos sociales habituales y sostenidos a través de las fronteras nacionales para su ejecución (Portes et. al., 2003: 18).

Tal noción se ha extendido en el marco de la ampliación de las nuevas tecnologías de la comunicación y el transporte que facilitaron la salida de los migrantes y les permitieron

9 Para un análisis sobre los precursores del transnacionalismo actual véase Portes et. al (2003) quien se remonta hasta la Europa Medieval en donde identifica enclaves extranjeros de mercaderes como un ejemplo temprano de transnacionalismo económico.

10 Lo que le ha hecho plantear a Portes et. al. (2003) [1999] que "los estudios acerca de la migración transnacional forman un campo emergente altamente fragmentado, al que todavía le falta rigor analítico y un marco teórico bien definido por lo que se requiere delimitar bien dicha noción". 
desarrollar un flujo continuo, bi (y multi añadiría) direccional no solo de información y recursos, sino también de afectos, sueños, ideas, imaginarios, problemas cotidianos, entre otros. En tal proceso se transformó tanto el carácter de las comunidades de origen como aquel de los lugares de asentamiento en el exterior (Portes, 2002:139).

Los autores que trabajan desde esta perspectiva plantean que este fenómeno está compuesto por un creciente número de personas que viven una doble vida: "hablan dos idiomas, tienen dos hogares en ambos países y su vida discurre en un contacto continuo y habitual a través de las fronteras nacionales y abarca una gama de actividades económicas, políticas y sociales" (Portes et. al., 2003: 15).

En términos de la teoría sociológica ello remite a la disolución de la relación comunidadsociedad con la que se estudió tradicionalmente las formas de organización social. En el espaciotiempo de la globalización compleja los seres sociales no encarnan únicamente relaciones primarias sustentadas en vínculos directos y relaciones secundarias fundadas en los roles de la vida pública sino que además se ven inmiscuidos en nexos sociales mediados por (nuevas) tecnologías comunicacionales y grandes organizaciones e industrias culturales que remiten a unos vínculos terciarios sostenidos en otra dimensión socio-espacial (Ramírez y Ramírez, 2005) ${ }^{11}$.

El transnacionalismo puede definirse entonces como "el proceso a través del cual los migrantes forjan y sostienen relaciones trenzadas que conectan entre sí diferentes sociedades de origen y asentamiento. Se denominan a estos procesos transnacionalismos con la idea de enfatizar que hoy construyen campos sociales que cruzan dimensiones geográficas, culturales, políticas, y económicas, pero donde el elemento esencia es la multiplicidad de relaciones que los 'transmigrantes' mantienen entre ambos polos, sociedades de origen y sociedades de destino" (Portes et al., 2003: 41).

En esta dirección Levitt (2001) plantea que los migrantes no viajan solos sino que van acompañados de ideas, comportamientos, identidades y capital social a los cuales denomina remesas sociales. "Son como los recursos sociales y culturales que los migrantes traen con ellos a los países que los reciben" (2001: 55). Estas remesas sociales (que juegan un papel central en el empoderamiento del migrante, en el desarrollo comunitario y en la integración política) son transformadas en el país receptor y transmitidas de vuelta a las comunidades expulsoras gracias a las redes y nodos que permiten hablar de comunidades transnacionales.

La emergencia de redes reproduce los efectos culturales entre los países de destino y los países expulsores, en donde emergen nuevos productos culturales. Arjun Appadurai (1996) analiza esta 'cultura de la globalización' en la que la imaginación trabaja como una fuerza social constitutiva del mundo actual. Sus efectos inmediatos se transfieren, por intermedio de flujos comunicacionales y redes transnancionales, a los procesos de conformación de nuevas identidades que se confrontan a las viejas identidades conformadas desde el Estado-Nación. La época actual de globalización está caracterizada así por la existencia de otras fuerzas gemelas que acompañan a la imaginación, sustentadas en los procesos de migración de masas y en la mediación electrónica ${ }^{12}$.

Estas fuerzas son materiales de construcción de lo que Beck (1998) denomina "mundos imaginarios" que personas y grupos en todo el mundo suministran, intercambian, y viven con significaciones diferentes. Involucran un proceso de construcción de la vida cotidiana en el espacio social de los migrantes y en diferentes lugares generando la emergencia de un espacio

11 Para un análisis del concepto de las relaciones terciarias, ver García Canclini (1997).

12 Ong (1999) critica a Appadurai quien se pregunta si la imaginación como una práctica social puede ser tan independiente de las estructuras nacionales, transnacionales y económico-políticas que permiten, canalizan y controlan los flujos de personas, cosas e ideas. Además señala que al ignorar la economía política de la comprensión tiempo espacial, da la apariencia engañosa de que todos pueden tener la misma ventaja de movilidad y de comunicación y de que el transnacionalismo ha fungido como liberador de todas las personas, tanto en términos espaciales como políticos. 
transnacional o lo que Besserer (2004) denomina topografia transnacional. Este es un fenómeno primordialmente social en el cual las cadenas y redes migratorias constituyen microestructuras que sostienen los movimientos de población en el tiempo y en el espacio configurándose una "representación de lugares y una interpretación propia que hacen los actores del espacio en el que sucede la acción” (Besserer, 2004: 21-22).

Así, este lente teórico sirve para entender el complejo campo de la migración, teniendo en cuenta las continuidades, variaciones y específicas características sociales y especiales de cada dinámica migratoria -es decir, sus relaciones con variables relativas a las características demográficas (género, edad, etc.), socio-económicas (pobreza, empleo), territoriales (rural, urbano) y tecnológicas (uso de NTICs) que van marcando diferencias en las formas y mecanismos de la migración.

Estas particularidades ha dado paso que dentro de la literatura de los estudios transnacionales se distinga varias formas y niveles de transnacionalismo utilizando nociones como el de comunidades transnacionales, espacio social transnacional, campo social transnacional, circuito migratorio, sistema migratorio transnacional, entre los principales.

Para efectos de este artículo interesa resaltar que las comunidades transnacionales de migrantes son grupos de personas que participan de forma rutinaria en un ámbito de relaciones, prácticas y normas que abarcan ambos lugares el de origen y de destino" (Glick Schiler et al., 1992). Kearney y Negengast (1989) pioneros en proponer esta noción al estudiar la migración mexicana a California señalan que este concepto debe entenderse en términos de componentes sociales, políticos y económicos internacionales, pero no en un sentido espacial y ciertamente no en el sentido de 'comunidad' que ha sido históricamente usado en la literatura de las ciencias sociales (Kearney y Negengast, 1989: 2).

De esta manera su crítica apunta a las visión dominante en las ciencias sociales, que ha definido la comunidad como un espacio delimitado y habitado por una población más o menos coherente con relaciones sociales continuas, un 'modo de vida compartido', y una serie internamente consistente de creencias, valores y reglas que gobiernan las vidas de todos (Kearney y Negengast, 1989: 1).

Besserer (1999) y posteriormente otros autores, al hacer un análisis sobre dicho concepto ponen la mirada en la noción de comunidad vista esta como una realidad fragmentada y coyuntural, enfatizando el estudio de múltiples narrativas que construyen a la comunidad como un calidoscopio. Al analizar los estudios 'empiristas' sobre comunidades trasnacionales los clasifican en tres sentidos de acuerdo a la definición que dan del mismo.

En primer lugar hay una noción de comunidad transnacional entendida como una comunidad que se extiende y consolida más allá de las fronteras. Desde esta perspectiva se hace referencia a una comunidad como transnacional cuando sus miembros han cruzado fronteras nacionales. Un segundo grupo se refiere a la comunidad transnacional vista como resultado de la construcción de la nación. A partir de esta mirada se da centralidad al proceso continuo de construcción del estado-nación en donde existe un cuestionamiento sobre el concepto de nación como producto acabado. Y el tercer enfoque que clasifica Besserer, hace alusión a las comunidades transnacionales entendidas como comunidades que se consolidan en un momento de 'desvanecimiento' del estado nación. El énfasis aquí se pone en el resquebrajamiento histórico de la preeminencia de la imagen del estado-nación como unidad política, cultural y social predominante ${ }^{13}$.

Esta perspectiva teórica dentro de los estudios migratorios también ha sido utilizada para entender la migración internacional ecuatoriana. Aunque la mayoría de estudio se han centrado en las zonas urbanas -de donde salió la mayoría de la población en la 'estampida migratoria' de

13 Existe una amplia literatura sobre comunidades transnacionales sobre todo para explicar la migración MéxicoEstados Unidos. 
fin de siglo- existen algunos trabajos sobre comunidades rurales, sobre todo realizados en las provincias de Cañar y Azuay lugares de donde salió la primera ola migratoria desde la década de los 50's.

Uno de estos trabajos son los realizados por Jokisch (1998) y Kayle (2000) y su posterior comparación (Jokisch y Kayle, 2005) quienes, desde una perspectiva transnacional, ven las transformaciones a largo plazo en las comunidades de Tomebamba, Biblicay y Quipal ubicadas en las provincias señaladas anteriormente cuyos habitantes han migrado a New York, Boston y Filadelfia.

Si bien dichos trabajos hacen un análisis riguroso de largo alcance de comunidades del austro ecuatoriano ${ }^{14}$, concluyen que el vaciamiento paulatino de las comunidades emisoras fruto de la migración internacional ha dejado tras de si "comunidades fantasmas" o "pueblos abandonados". Los autores señalan que se produjo una cadena migratoria que "lentamente separó de la comunidad a la mayoría de sus habitantes anteriores. Estas comunidades se caracterizaban en los años ochenta, por ser solo de mujeres y niños, y, en los noventa, sólo de niños y abuelas. En la actualidad, en estas comunidades un número notable de abuelos también han partido" (Jokisch y Kyle, 2005: 63-64).

Más allá de que señalan la existencia de una mayor complejidad social fruto de la migración trasnacional, para Jokisch y Kyle la comunidad existe y la circunscriben solamente a un espacio local y a un territorio limitado y, por otro lado, siguen analizando en términos de comunidades emisoras y receptoras. En otras palabras, no logran superar la visión dicotómica, la manera convencional de definir el espacio y la propia comunidad. Es de esta visión reduccionista del que es necesario tomar distancia.

Como hemos visto ciertos antropólogos y pensadores de la migración con enfoque transnacional dieron nuevas perspectivas de análisis para dejar de ver en términos duales como comunidades emisoras y comunidades receptoras, y quizá constituya uno de los grandes aportes a la teoría social ${ }^{15}$. Como se indicó, fruto de la movilidad multidireccional y de los vínculos que mantienen los migrantes y no migrantes en al menos dos países, se empezó hablar de procesos transnacionales. Kearney $(1991,1996)$ señalaba que algunas comunidades están transformándose en entidades cada vez más dispersas y menos identificables a un solo espacio y estas persisten bajo la forma de comunidades transnacionales 'desterritorializadas'. Gupta y Ferguson (1997) al poner la mirada en los sujetos, señala que están constituyendo sus propios espacios sociales de una manera diferente a los modos convencionales y los están 'reterritorializando'.

Para el caso que he analizado de Pepinales, utilizo el término re-desterritorialización para indicar por un lado la ruptura de la ecuación -en términos naturales y esenciales- entre comunidad y territorio, pero a su vez indicar la parcial, temporal, cíclica o virtual resignificación y recomposición de la comunidad en varias topografías.

Con este énfasis lo que proponemos, lo que interesa entender y analizar es la idea que los habitantes de este lugar tienen sobre Pepinales, sobre su comunidad, a partir del análisis de sus prácticas, de sus interacciones y de los procesos de construcción de sus redes sociales. En este entretejido, indudablemente que cada lugar donde migraron tanto interna como internacionalmente, ocupa un lugar diferente, pero juntas forman un todo.

14 Otra de las comunidades bastante estudiadas dentro del enfoque transnacional es la de los Otavaleños, vistos estos como comerciantes transnacionales. Los trabajos de Kyle (1999, 2000), Meisch (2002), Maldonado (2004), Ruiz (2006), Ordóñez, (2008) así como las permanentes citas de Portes (1996a, 1996b, 1997a, 1997b) constituyen referencias indispensables para entender la conformación de este grupo étnico en el escenario global. La particularidad del caso otavaleño es que ellos no se autodefinen como migrantes, sino como comerciantes que históricamente han salido fuera de su comunidad a comercializar sus productos. Otro de los grupos indígenas también estudiados en Ecuador son los Saraguros. Sobre ellos ver Belote et. al (2005) y Cruz (2008).

15 De igual manera los teóricos transnacionales proponen re pensar y reformular no solo el concepto de comunidad sino la noción de frontera, redes sociales, cultura, identidad o ciudadanía. Para un análisis sobre estos aspectos ver Garduño (2003), Levitt y Glick Schiler (2006). Por el otro lado, también han surgido varias críticas a estas entradas teóricas entre las que se puede señalar la invisibilidad en estos estudios de las personas no-migrantes, el no tener en cuenta las relaciones asimétricas y de poder o la propia 'reificación' de los conceptos. 


\section{Pepinales, tierra querida...}

Para llegar a Pepinales desde Quito se tiene que ir a Riobamba y de ahí tomar un bus rumbo a Alausí. En dicha ciudad hay que agarrar otro bus o una camioneta que vaya rumbo a Huigra. Casi a mitad de camino, pasando Sibambe, se encuentra la entrada al recinto. Las camionetas y buses paran en una caseta donde hay un cartel que señala la entrada al pueblo. No existe trasporte público que llegue al centro o a las partes altas, salvo que se pague un flete hacia estos lugares.

El camino es de segundo orden, lastrado y polvoriento. Al caminar los primeros metros se visibilizan las primeras casas las mismas que se encuentran dispersas, separadas por terrenos algunos sembrados de maíz y otros potreros con vacas pastando. Varias de las viviendas parecen cerradas. A primera vista se ven dos diferentes tipos de construcción: unas viejas de adobe y bareque y otras nuevas de concreto. Muchas dan la impresión de estar cerradas, pero la presencia de gallinas, cerdos y ropa aun mojada en los tendederos nos avisan que alguien vive en su interior.

Al seguir caminando percibo un silencio en el ambiente solo interrumpido por el mugido de alguna vaca y unos perros que ladran al escuchar mis pasos. Me encuentro con dos personas en el camino y les pregunto dónde está la iglesia, me responden que un poco más adelante. Al llegar allá me cruzo con un señor de edad avanzada que salía de la iglesia, sentí que se asombró al verme. Le saludo y comento que vi unas fotos de Pepinales por Internet y que me dio ganas de venir a conocer. Don Leo me respondió que ahora ya viven pocas personas porque la mayoría ha salido a afuera, pero que en fiestas se pone 'llenecito' el pueblo.

Mientras caminamos juntos me cuenta que la mayoría de la gente que allí vive se dedica principalmente a la ganadería y también a la agricultura. Que siembran maíz, trigo, cebada, papa, lenteja, pero que casi todo es para ellos mismos y algo para la venta. Décadas pesadas la agricultura era la principal actividad, por lo que se conocía a Pepinales como 'el granero de Alausí'. Sin embargo, desde mediados de los ochenta, se produjo una disminución de la actividad agrícola en las haciendas Cusunag y Yalancay donde muchos laboraban. Según me contaron varias personas, las tierras empezaron a decaer, ya no eran tan fértiles sobre todo por la introducción de químicos y pesticidas, sumados a los cambios climáticos, sequías que condujeron a la larga a que la agricultura sea solamente para el autoconsumo.

Este factor del decaimiento de actividad agrícola fue uno de los causales centrales para que la gente empiece a salir de Pepinales produciéndose una migración tanto interna (rural-rural como rural-urbano) como internacional desde la década de los setentas ${ }^{16}$. En efecto, si uno mira la estructura piramidal de la población que actualmente reside en Pepinales, se observa claramente que es una comunidad habitada fundamentalmente por menores y ancianos. En el centro de la pirámide (tabla No. 1), que corresponde a la población económicamente activa y reproductiva, se puede observar que se encoge en relación a sus polos. Es sobre todo este segmento de la población, correspondiente a las edades entre 25 y 50 años de edad de ambos sexos, la que se encuentro viviendo fuera del recinto, tanto en el extranjero, pero también en otros lugares dentro del Ecuador. Del censo realizado en el 2006, el 37\% viven aun en Pepinales, un porcentaje similar en otros lugares del Ecuador y el $26 \%$ radican fuera del país ${ }^{17}$.

16 A este factor hay que señalar otros como el hacinamiento, falta de tierras, trabajos temporales a la costa, estudios, deudas, cercanía con localidades de alta migración como Chunchi, Biblián, Tambo, formación de cadenas y redes migratorias y conformación de un habitus migratorios, que explican la multicausalidad de la migración de los pepinalenses.

17 El trabajo de campo se realizó entre el 2006 y 2008 utilizando la propuesta metodología multilocal (Marcus, 1995) y el análisis de topografías transnacionales (Besserer, 2004) utilizando varias herramientas tales como: observación participante, entrevistas, historias de vida, censo, genealogías, trabajo de archivo. 


\section{Tabla 1. Pinjmide poblactoral de Pephahes}

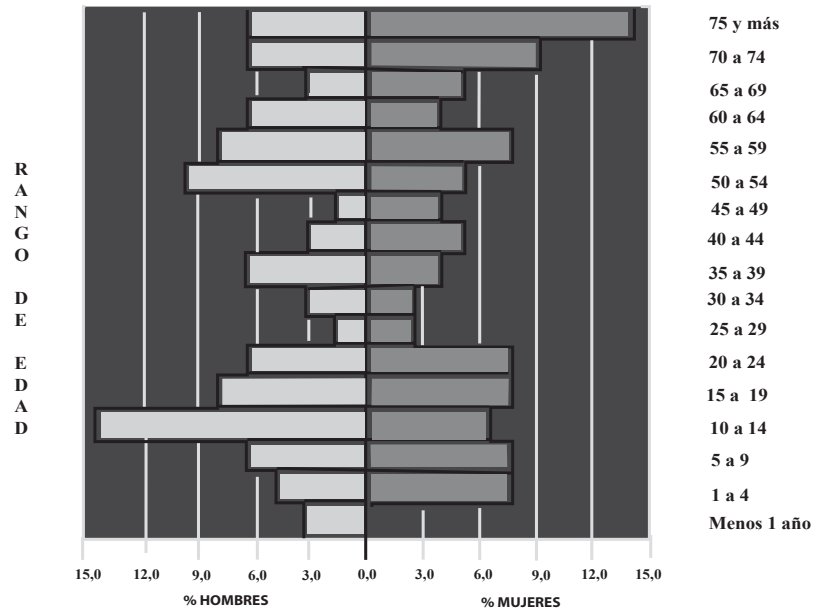

Elaborado por el autor

Lo que para los investigadores podría representar porcentajes que hablarían de alta migración y/o de 'pueblos fantasmas' (que incluso, como señala don Leo, es todo lo contrario en épocas de fiestas), los pepinalenses han construido una geografía colectiva de su comunidad que rompe con la visión clásica de entender a las mismas.

\section{'Los pepinalenses estamos por todo lado'}

Al interrogar sobre los lugares donde han migrado o vivido de manera temporal o estacionaria, un primer listado de todos los lugares individuales nombrados por los pepinalenses recogió 66 localidades en diez países. Sin embargo, lo que interesa en la metodología utilizada es la geografía colectiva de la comunidad, es decir, aquellos lugares que fueron nombrados más de una vez y que no sólo son compartidos por los miembros de una familia, por lo cual el número se redujo a menos de la mitad.

A nivel nacional se produjo en un primer momento (1975-1980) una migración rural-rural asentándose en lugares como Cutyguay, Pagma y Santa Rosa. En estos tres lugares todavía radican varios pepinalenses. Posteriormente (1981-1985) hubo una movilidad muy fuerte a las zonas urbanas sobre todo de jóvenes pepinalenses que salieron hacia Alausí, Riobamba y Quito.

Estos desplazamientos al interior del país rural-rural y rural urbano, constituyen la antesala de la migración internacional y muchas de estas fueron utilizadas como ciudades intermedias antes de su viaje al exterior. Este es un momento donde se empiezan a tejer las redes comunitarias desterritorializadas sin las cuales no es posible entender el proyecto migratorio hacia el exterior.

En efecto, a partir de mediados de los ochenta se empiezan a dirigir hacia Alemania, específicamente a la ciudad de Bonn dando inicio al 'boom' migratorio de pepinalenses a 
dicho país. En las narrativas de vida, hasta la actualidad está presente este evento que forma parte de la memoria colectiva de la comunidad y que, con el pasar de los años, posibilitó la formación de cadenas y redes migratorias. En efecto, más de veinte años después de que salieron los tres migrantes pioneros que "abrieron el paso" -y cuyos nombres son recordados con exactitud por los habitantes-, existen todavía, aunque escasos, flujos migratorios que continúan dirigiéndose hacia este lugar.

A principios del nuevo siglo cuando se refuerzan controles migratorios y se endurece la política migratoria en dicho país, muchos que vivían y viven 'sin papeles' fueron expulsados de Alemania motivo por el cual algunos optaron por regresar a Ecuador (y muchos se radicaron en Quito, Riobamba, Alausí y en menor medida en Pepinales) y otros re-emigraron hacia España.

A diferencia de lo que ocurrió en Alemania, país en el que la concentración se dio en una sola ciudad (Bonn), en España en un inicio la mayoría se radicó en Madrid, en especial en el barrio de Moratalaz, pero en la actualidad hay pepinalenses en la región de Murcia/Lorca, Logroño y en menor medida en ciudades como Barcelona, Valencia y Pamplona.

Finalmente, si bien los estados receptores han tratado de reafirmar su soberanía y reducir la migración indocumentada poniendo obstáculos a la movilidad geográfica de los migrantes (Sassen, 1996) estas políticas no han detenido la conformación de redes transnacionales. Ya es un lugar común dentro de los estudios migratorios contemporáneos señalar la importancia y el papel que han cumplido las nuevas tecnologías de información y comunicación (NTICs) para el mantenimiento de los vínculos entre el "aquí" y el "allá".

Sin embargo, la posibilidad de entablar relaciones perdurables y cotidianas por medio de la comunicación vía computadora, sin mediar el contacto físico, está promoviendo y sosteniendo nuevas relaciones sociales y posibilitando la formación de un nuevo tipo de comunidades: las “comunidades virtuales” y cotidianidades 'on line' (Ramírez, 2007). Para el caso aquí analizado, los pepinalenses que se encuentran en el exterior están conectados a través de su portal www. pepinales.com, el cual apunta a reforzar los lazos comunitarios, de pertenencia e identidad.

\section{Mapa 1. Pephyales ne-desterritorinlizado 1975-2009}

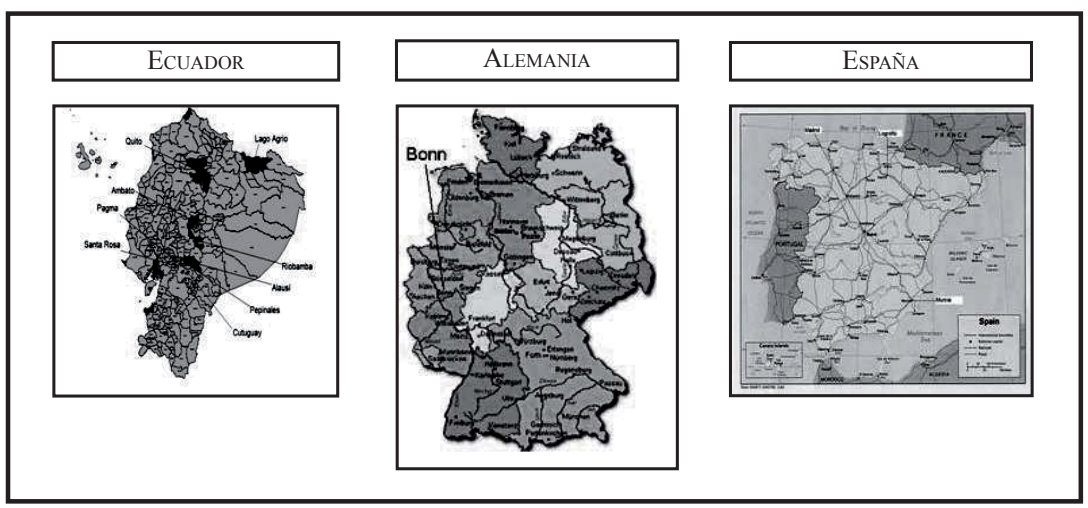

Fuente: www.pepinales.com 


\section{Cierre: la comunidad como un archipiélago}

Este breve ejercicio de rastrear la noción de comunidad ha servido para ejemplificar y alertar cómo ciertos conceptos pueden quedar obsoletos a la luz de las nuevas configuraciones sociales que se han producido en un mundo cada vez más globalizado.

Pero más allá de modas intelectuales o de importación de marcos teóricos, es necesario recordar que es a partir del quehacer etnográfico -lo que define al antropólogo y la disciplinadesde el cual debemos re-pensar y re-elaborar los conceptos.

Para concluir quiero dejar señalado solamente algunas de las consideraciones teóricas fruto del trabajo de campo realizado en las diferentes topografías donde han migrado y, por su puesto, en Pepinales. En primer lugar, no se puede entender la comunidad sin la noción de pertenencia, entendiendo por pertenencia, siguiendo a Cohen (1982), como algo que va mucho más allá de haber nacido (y añadiría permanecido) en un lugar ${ }^{18}$.

En segundo lugar, en contextos de alta migración esta comunidad ampliada redesterritorializada la entiendo como un "archipiélago activo translocal" en permanente cambio, en la cual cada isla no solamente constituye un lugar donde se asientan los miembros de un lugar de origen particular, sino que también y sobre todo constituyen capitales sociales que poseen los individuos ${ }^{19}$.

Como vimos Pepinales, como varios lugares de Ecuador, ha experimentado un proceso de migración tanto interna (rural-rural y rural-urbano) como internacional. Si bien no puede negarse que han existido cambios demográficos fruto de la movilidad de sus habitantes, no se puede hablar de una desintegración, ni de vaciamiento o surgimiento de "comunidades fantasmas". Como plantea Hobsbawn (2003) es la dificultad de los investigadores de encontrar lo que tradicionalmente conocemos por comunidad, lo que ha hecho que su uso sea indiscriminado y vacío.

Por tal motivo, sólo investigaciones de tipo longitudinal y multisituadas que den cuenta de la movilidad de las personas, sentidos de pertenencia, actividades y condiciones laborales, lúdicas, festivas, formación de nuevas organizaciones, estatus migratorio, entre otros tópicos, nos darán información más certera sobre los cambios y continuidades que han experimentado las comunidades en los contextos migratorios y cómo tenemos que empezar a encontrarlas, entenderlas y re definirlas.

Luego del trabajo etnográfico y de analizar detenidamente los movimiento migratorios de cuatro décadas de una comunidad rural se puede afirmar que esta se ha re-desterritorializado, termino que utilizo para entender a la comunidad en movimiento y no anclada a un solo lugar. En este proceso primero se desterritoraliza, pero conforme se van asentando y solidificando algunas topografías se inicia una parcial, temporal o virtual re-significación y re-composición de la comunidad en dichos lugares. De esta manera la comunidad se re-desterritorializa.

Este proceso produce la conformación de una nueva geografía colectiva de la comunidad, teniendo las nuevas áreas diferente importancia, papel y significado. Como indicamos anteriormente, se pudo ubicar más de sesenta localidades donde han migrado pero solo algunas adquieren centralidad e importancia en la colectividad.

18 Este autor sugiere que "una persona es una pieza integral del maravillosamente complicado tejido que constituye la comunidad; esa persona es una depositaria de su cultura, orgullosamente distinta y conscientemente preservada -un depósito de su tradición y de sus valores, un ejecutante de sus sacras destrezas, un experto en sus expresiones e idiosincrasias. La profundidad de esa pertenencia se revela en las formas de organización social y asociación en la comunidad, de tal modo que cuando una personas es identificada como perteneciente a un grupo particular de parentesco o barrio, se convierte al mismo tiempo, en un miembro reconocible de la comunidad en su conjunto y de su variedad cultural" (Cohen, 1982: 21).

19 Por capital social entiendo, siguiendo a Bourdieu y Wacquant (1992), como "la suma de recursos, reales o virtuales, que devenga una red perdurable de relaciones más o menos institucionalizada de conocimiento y reconocimiento mutuo". 
Los casos de re-desterritorialización, permiten ejemplificar tipos de asentamientos translocales y nacionales que no solo conectan dos estados nacionales fronterizos (como han sido analizados, por lo general, los estudios de migración transnacional), conecta la movilidad interna con la(s) internacional(es) y permite entender a la comunidad como un espacio geográfico socialmente vivido por sus miembros, donde ellos mismos han resignificado su sentido de pertenencia comunitaria según la 'isla', 'islote' o 'piedra' en la que habitan, en la cual cada una tiene su propia dinámica pero en su conjunto forman un archipiélago activo en movimiento.

La metáfora de archipiélago activo ${ }^{20}$ es valiosa porque al entender la movilidad como una estrategia y forma de vida, esta puede dar paso a que algunas topografías tengan mayor o menor centralidad, decaigan o que a futuro aparezcan nuevas.

Es por esto que si bien considero útil y valioso los aportes de los diferentes autores que utilizaron la noción de comunidad transnacional, esta incluso llega a ser estrecha. Es muy útil al momento de ver las conexiones, los lazos, interacciones entre 'el aquí' y 'el allá', que también se puede explicar analíticamente a partir del enfoque de las redes sociales, pero pierde fuerza explicativa al momento de entender lo que sucede en cada una de las 'islas' que se asentaron.

De esta manera cada 'isla' tiene su propia dinámica y particularidad, pero evidentemente conocen y están al tanto de lo que sucede en las otras. Los hallazgos del trabajo permiten hablar de una comunidad en el cual no solo mantienen el vínculo entre el lugar de origen y el de destino (que tuvo su mayor intensidad en los inicios de la migración) sino también, y de manera más regular, entre destino y destino, ayudados ahora por las nuevas tecnologías, pero en dicho vínculo el lugar de origen sirve de ancla para pensar, imaginar y vivir en comunidad.

\section{Bibliografía}

Aguirre Beltrán Gonzalo. 1973, Regiones de Refugio. El desarrollo de la comunidad y el proceso dominical en mestizoamérica, INI, México.

Arjun, Appadurai. 1996, Modernity at Large Cultural Dimensions of Globalization, Public Worlds Series, Vol. 1 Cowpublished with Oxford University Press.

Basch, L., N. Glick Schiller y C. Szanton Blanc (eds.) 1994, Nations Unbound: Transnational projects, Postcolonial Predicaments, and Deterritorialized Nation-States, Ginebra.

Beck, Ulrich. 1998, ¿Qué es la globalización? Falacias del globalismo, respuestas a la globalización. Editorial Paidós, Barcelona.

Belote, Linda y Jim Belote. 2005, “¿Qué hacen dos mil saraguros en EE.UU. y España?”, en: Herrera G. et al., La migración ecuatoriana. Transnacionalismo, redes e identidades, FLACSO - Plan Migración Comunicación y Desarrollo, Quito.

Besserer, Federico. 2004, Topografias transnacionales. Hacia una geografia de la vida transnacional, UAM, PyV editores, México.

1999, "Estudios transnacionales y ciudadanía trasnancional", en: Gail Mummert (ed), Fronteras Frangmentadas, Michoacán, COLMICH, México, pp. 215-238.

Bourdieu, Pierre y Loic Wacquant. 1992, An Invitation to Reflexive Sociology. Universidad de Chicago, Chicago.

Clifford, James.1999, Itinerarios trannculturales, Gedisa, Barcelona.

Cruz Zúñiga, Pilar. 2008, "Comunidades transnacionales indígenas: experiencia migratoria del pueblo saraguro en Vera (España)", en: Torres, Alicia y Jesús Carrasco (Coords.) Al filo de la identidad. Migración indígena en América Latina. FLACSO; UNICEF; ALISEI, Quito.

20 John V. Murra (1996), al analizar la organización andina precolonial, fue el pionero en utilizar el concepto de "archipiélago" para dar cuenta del manejo de los territorios étnicos y del control de los pisos ecológicos. 
Cohen, Anthony. 1982, Belonging. Identity and Social Organisation in British Rural Cultures, Universidad de Manchester, Manchester.

Delgado, Manuel. 2005, "Espacio público y comunidad”; en: Lisbona (ed.), La Comunidad al debate. Reflexiones sobre el concepto de comunidad en el México contemporáneo. El colegio de Michoacán - Universidad de Ciencias y Artes de Chiapas. México.

Delanty, Gerard. 2006, Community. Comunidad, educación ambiental y ciudadanía. Editorial Graó, Societat Balear d'Educació Ambiental y Societat Catalana d'Educació Ambiental, Barcelona.

García, Fernando. 1980, "Introducción”, en: Revista de la Universidad Católica, Vol. 8, No. 26, Quito.

Garduño, Everardo. 2003, "Antropología de la frontera, migración y procesos transnacionales", en: Frontera Norte, Vol.15, Julio-Diciembre, Colegio de la Frontera Norte, México.

Goldring, Luin. 1992, "La migración México-EUA y la trasnacionalización del espacio político y social: perspectivas desde el México rural”, en: Estudios Sociológicos X, 29, México, pp. 315-340.

Glick Shiler, Nina, L. Basch y C Szanton-Blanc. 1992, "Transnatrionalism: A new Analytic Framework for Understanding Migrations", en: Glick Shiler, Nina, L. Basch y C Szanton-Blanc (comp.) Towards a Transnacional Perspectiva on Migration: Race, Class, Ethnicity, and Nationalism Reconsidered, New York Academy of Sciences, New York, pp. 1-24

Gupa, Akhil y James Ferguson. 1997, "Beyound 'Culture': Space, Identity, and the Politics of Difference”, en: Gupta y Ferguson (eds.) Culture, Power, Place: explorations in Cultural Anthropology, Durke University Press, Durham, pp 33-51.

Hobsbawm, Eric. 2003, Años interesantes: una vida en el siglo XX. Crítica, Barcelona.

Kearney Michael y Carole Negengast. 1989, Anthropological Perpectives on Trasnational Communities in Rural California, Working Group on Farm Labor and Rural Poverty Working Paper No.3, California Institute for Rural Sudies, Davis.

Kearney, Michael. 1996, "Reconceptualizing the Peasantry", en: Antropology in Global Perspective. Boulder, Westview Press, Inc.

1991, "Borders and Boundaries of State and Self at the End of the Empire", en: Journal of Historical Sociology, Vol. 4, marzo. Kyle, David. 2003 [1999], "La diáspora comercial de Otavalo", en: A. Portes, L Guarnido y P Landolt (coord), La Globalización desde abajo: transnacionalismo inmigrante y desarrollo. La experiencia de Estados Unidos y América Latina, Flacso-México / Porrua, México, pp. 315-351.

1986, "From the Invisible Hand to Visible Feet: Anthropological Studies of Migration and Development", en: Annual Review of Anthropology, No. 15.

Kyle, David. 2000, Transnational Peasants: Migrations, Networks, and Ethnicity in Andean Ecuador, The Johns Hopkins University Press, Baltimore and London.

Levitt Peggy. 2001, The Transnacional Villagers, Univ. of California. Press, Berkeley, L.A.

Levitt, Peggy y Nina Glick Schiller. 2006, "Perspectiva internacional sobre migración"; en: Portes y DeWind (coords.), Repensando las migraciones. Nuevas perspectivas teóricas y empíricas. Porrua - U. Zacatecas INM, México.

Lisbona, Miguel. 2005, La Comunidad al debate. Reflexiones sobre el concepto de comunidad en el México contemporáneo. El colegio de Michoacán - Universidad de Ciencias y Artes de Chiapas, México.

Jokish, Brad. 1998, Landscape of Remittances: Migration and Agricultural Change in the High lands of South Central Ecuador, Ph.D. Graduate School of Geography, Clark University, Worcester, MA.

Jokish, B. y Kyle. D. 2005, "Las transformaciones de la migración del Ecuador 1993-2003", en: Herrera G, C. Carrillo y A. Torres (eds.), La migración ecuatoriana. Trasnancionalismo, redes e identidades, FLACSOECUADOR y Plan Migración Comunicación y Desarrollo, Ecuador.

Maldonado, Gina. 2004, Comerciantes y viajeros. de la imagen etnoarqueológica de "lo indígena" al imaginario del Kichwa Otavalo "universal", FLACSO - Sede Ecuador : Abya - Yala, Quito.

Marcus, George. 1995, "Ethnography in/of the World System. The emergence of Multi-Sited Ethnography", en: Annual Review of Anthropology, No. 24, pp. 95-117.

Martínez, Carmen. 2007, "De militantes, religiosos, tecnócratas y otros investigadores: la antropología ecuatoriana y el estudio de lo indígena desde la década de los setenta", Mimeo, FLACSO-Ecuador, Quito.

Martínez, Luciano. 2002, Economía Política de las comunidades indígenas. Ecuador, ILDIS - Abya-Yala OXFAM - FLACSO, Quito.

Meisch, Lynn. 2002, Andean Entrepreners: Otavalo Merchants and Musicians in the Global Arena, Universidad de Texas, Austin. 
Miles, Ann. 2004, From Cuenca to Queens. An Anthropological Story of Transnational Migration, Univesity of Texas, Austin.

Murra, John. 1996, "Valorización de la diversidad ecológica. El control vertical de un máximo de pisos ecológicos y el modelo en Archipiélago", en: Morlon, Marlon Pierre (Comp.) Comprender la agricultura campesina en los Andes centrales: Perú-Bolivia, Institut français d'études andines. IFEA - Centro Bartolomé de las Casas, Lima.

Ong Aihwa. 1999, Flexible citizenship: The cultural logia of transnationality, Duke University Press

Ordónez, Angélica. 2008, "Migración transnacional de los kichwa-otavalo y la fiesta del Pakwar Raymi" en: Torres, Alicia y Jesús Carrasco (eds.) Al filo de la identidad. Migración indígena en América Latina. FLACSO; UNICEF; ALISEI, Quito.

Parsons, Talcon. 1999, El sistema social. Editorial Alianza, Madrid.

Portes, Alejandro. 2002, "Inmigrantes, claves para un futuro inmediato", en: Cuadernos Étnicas, Universidad de Jaén, España.

1997, Neoliberalism and the Sociology of Development Review, vol. 23, No. 2, pp. 229-259

1997b, "immigration Theory for a New Century: Some Problems and Opportunities", en: International Migration Review, vol.31, No.4, pp.799-825

1996a, "Global Villagers: The Rise of Transnational Communities", en: The American Prospect, vol. 25, marzoabril de 1996, pp. 74-77.

1996, "Transnacional Communities: Their Emergence and Significance in the Contemporary World-Sistem", en: R.P Korzeniewidcz y W.C. Smith (comps.), Latin American in the World Economy, Greenwood Press, Westport, pp. 151-168.

Portes, Alejandro, Guarnizo Luis, Patricia Landolt. 2003 [1999], "El estudio del transnacionalismo: peligros latentes y promesas de un campo de investigación emergente", en: A. Portes, L Guarnizo y P Landolt (coord), La Globalización desde abajo: transnacionalismo inmigrante y desarrollo. La experiencia de Estados Unidos y América Latina, Flacso-México / Porrua, México, pp.15-44.

Portes, Alejandro y Josh DeWind. 2006, Repensando las migraciones. Nuevas perspectivas teóricas y empíricas, Porrua-U.Zacatecas-INM, México.

Ramírez Franklin y Ramírez Jacques. 2005, La estampida migratoria ecuatoriana (1997-2003): crisis, redes y repertorios de acción, CIUDAD-ABYA-YALA, Quito.

Ramírez, Jacques. 2010, Dónde está la comunidad?: migración ecuatoriana a España y Alemania, El caso de Pepinales, Tesis Doctoral en Antropología Social, Universidad Iberoamericana, México (inédito).

2009, "Ecuador migrante: redes, repertorios de acción y espacio social transnacional", en: Migraciones Contemporáneas. Contribución al debate, CIDES-UMSA, La Paz, Bolivia.

2007, “Aunque se fue tan lejos nos vemos todos los días: Migración y uso de NTIC", en: Los Usos de Internet: Comunicación y Sociedad, Tomo 2, FLACSO-ECUADOR-IDRC, Quito.

Redfield, Robert. 1930, Tepoztlán, a Mexican Village. A Study of Folk life. Chicago, Univesrsidad de Chicago.

1941, The Folk Culture of Yucatan. Chicago, Univesrsidad de Chicago.

1955, The Little Community. Chicago, Univesrsidad de Chicago.

Sánchez Parga, José. 2002, Crisis en torno al Quilotoa: Mujer, cultura y comunidad. Quito, CAAP.

Rouse, Roger. 1991, "Mexican Migration and the Social Space of Postmodernism", en: Diáspora, Vol.1, pp. 8-23.

Ruiz, Andrea. 2006, "Espacios de poder transnacional: Diferencias en la migración kichwa-otavalo", en: Rosa Santibáñez y Maiztegui Concepción (eds.) Inmigración: miradas y reflejos, Universidad de Deusto, España.

Sassen, Saskia. 2003, Los espectros de la globalización. Fondo de Cultura Económica, Argentina.

Stolen, Kristi Anne. 1987, A media voz: relaciones de género en la sierra ecuatoriana. CEPLAES, Quito.

Tönnies, Ferdinand. 1979, Comunidad y asociación. Editorial Península, Madrid.

Zárate, Eduardo. 2005, "La comunidad imposible. Alcances y paradojas del moderno comunalismo", en: Lisbona (coord.): La Comunidad al debate. Reflexiones sobre el concepto de comunidad en el México contemporáneo. El Colegio de Michoacán - Universidad de Ciencias y Artes de Chiapas, México. 